\title{
SOME OBSERVATIONAL APPEARANCES OF \\ $m=1$ DENSITY WAVES IN Be STAR DISKS
}

\author{
ATSUO T. OKAZAKI \\ College of General Education, Hokkai-Gakuen University, \\ Toyohira-ku, Sapporo 062, Japan.
}

\section{One-Armed Oscillation Model}

One-armed (i.e., $m=1$ ) density waves are the only global waves in nearly Keplerian disks (Kato 1983). Their frequencies are much smaller than the angular frequency of disk rotation. Based on this theory, Okazaki (1991) proposed that the long-term $\mathrm{V} / \mathrm{R}$ variations of $\mathrm{Be}$ stars are phenomena caused by the global $m=1$ oscillations in the equatorial disks. Hummel and Hanuschik (1993) showed that line profiles of disks with $m=1$ perturbation patterns are in agreement with the observed $V / R$ variability.

\section{Some Effects of $m=1$ Density Waves on Disk Emission}

We assume that the perturbation pattern is similar to the linear eigenfunction of the fundamental $m=1$ mode. The unperturbed disk extends up to 10 stellar radii and has the radial density distribution proportional to $r^{-3}$. The central star is a B0 main-sequence star.

Figure 1 shows an example of shell line variability. Notice that a hump which appears at the bottom of the profile shifts redward when $V / R>1$, and shifts blueward when $V / R<1$. Similar features are observed in the Balmer line profiles of EW Lac obtained by Kogure and Suzuki (1984). This suggests the presence of $m=1$ density waves in EW Lac envelope.

Figure 2 shows the correlation between the continuum variability and the $\mathrm{V} / \mathrm{R}$ variation for a disk with the $m=1$ perturbation pattern. In figure 2 we find that the continuum variability and the $V / R$ variation have the same period, but their phases are different by 0.25 (i.e., $90^{\circ}$ ). Whether the phase of the continuum variability is in advance of or in arrears of the $V / R$ phase depends on the sign of the eigenfrequency and the detailed form of the eigenfunction.

\section{References}

Hummel, W., and Hanuschik, R.W.: 1993, these proceedings

Kato, S.: 1983, Publ. Astron. Soc. Japan 35, 249

Kogure, T., Suzuki, M.: 1984, Publ. Astron. Soc. Japan 36, 191

Okazaki, A.T.: 1991, Publ. Astron. Soc. Japan 43, 75 


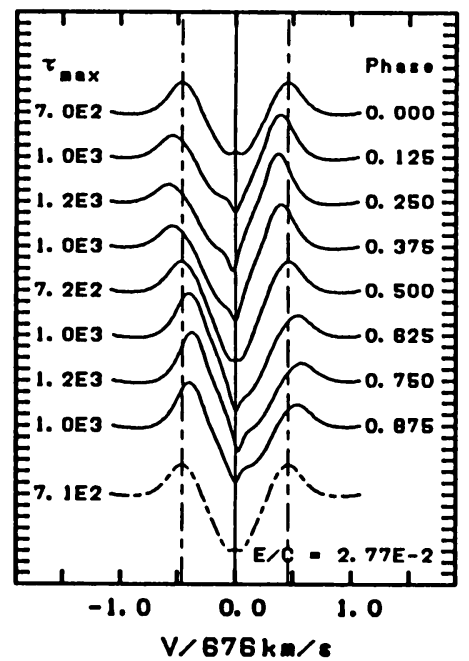

Fig. 1. Variability in a shell profile caused by the $m=1$ perturbation pattern. The solid profiles denote the profiles at the different phases and the dash-dotted profile denote the profile of the unperturbed disk. The vertical dash-dotted lines represent the peak velocities of the unperturbed profile. For each profile, the line optical depth, $\tau_{\max }$, is given. Also given is the ratio of the peak intensity to the continuum level, E/C.

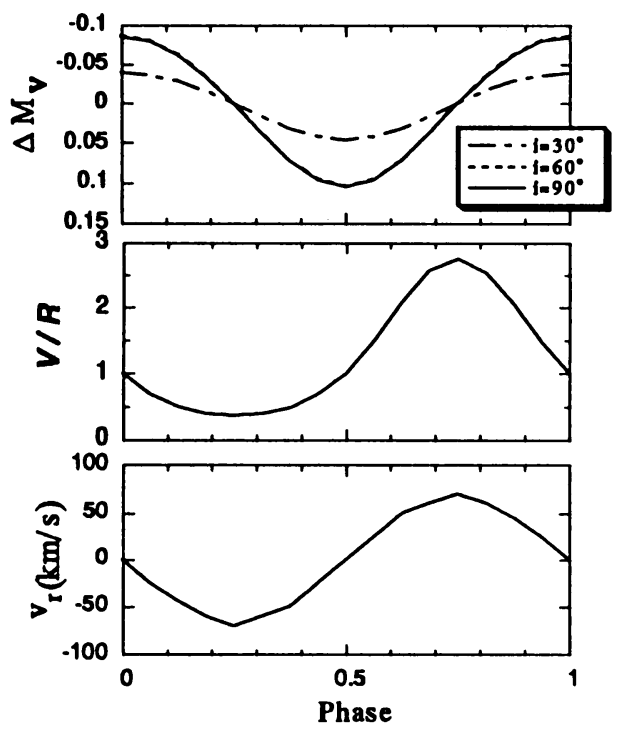

Fig. 2. The correlation between the continuum variability and the $V / R$ variation. The panels exhibit, from top to bottom, the magnitute of the continuum variability, the $V / R$ ratio, and the radial velocity, respectively. In the top panel, the solid, the dashed, and the dash-dotted curves denote variabilities for $i=90^{\circ}, 60^{\circ}$, and $30^{\circ}$, respectively. 